\title{
Investigation of Boundary Effects on the Natural Cavitating Flow around a 2D Wedge in Shallow Water
}

\author{
Xin Chen, ${ }^{1,2}$ Ying Chen, ${ }^{1,2}$ Jia-Yi Cao, ${ }^{1,2}$ and Jie $\mathrm{Li}^{1,2}$ \\ ${ }^{1}$ Department of Engineering Mechanics, Shanghai Jiao Tong University, Shanghai 200240, China \\ ${ }^{2}$ MOE Key Laboratory of Hydrodynamics, SJTU, Shanghai 200240, China
}

Correspondence should be addressed to Xin Chen, xinchen@sjtu.edu.cn

Received 8 July 2011; Accepted 31 October 2011

Academic Editor: Antonio Munjiza

Copyright ( $) 2011$ Xin Chen et al. This is an open access article distributed under the Creative Commons Attribution License, which permits unrestricted use, distribution, and reproduction in any medium, provided the original work is properly cited.

\begin{abstract}
When a cavitated body moves in shallow water, both flexible free surface and rigid bottom wall will produce great influence on the cavity pattern and hydrodynamics to change the motion attitude and stability of the body. In this paper, a single-fluid multiphase flow method coupled with a natural cavitation model was employed to study the effects of two kinds of boundaries on the natural cavitating flow around a two-dimensional symmetry wedge in shallow water. Within the range of the cavitation number for computation $(0.05 \sim 2.04)$, the cavity pattern would be divided into three types, namely, stable type, transition type and wake-vortex type. The shape of the free surface is fairly similar to that of the cavity's upper surface with well right-and-left symmetry. However, when the immersion depth and the cavitation number are decreasing, the symmetry of the cavity shape is destroyed due to the influence of bottom wall effects. When the cavitation number is less than about 0.1 , with the immersion depth going down, free surface effects exerts a stronger influence on the drag coefficient of this 2D wedge, whereas wall effects bring a stronger influence on the lift coefficient.
\end{abstract}

\section{Introduction}

Recently, high-speed underwater vehicles such as hydrofoilassisted crafts, fast ferries gain much interest in marine transport industry. When fast vehicles covered with supercavity navigate in shallow water like littoral zones, both the flexible free surface and the rigid water bottom will produce significant influence on the cavity pattern and the hydrodynamics of the vehicle. In such case, the so-called "boundary effects" will make the flow blocked and affect the motion attitude and stability of the vehicle.

Concerning the boundary effect problems caused by solid wall, many researches have been conducted so far in the world through the approaches of theoretical analysis, numerical simulation, and experimental investigation. Birkhoff et al. [1] theoretically studied the blocked cavitating flow around no-lift wedge and found that the defined drag coefficient in conventional experiments was very sensitive to the distance between the tunnel wall and the test model. Brennen [2] employed the Riabouchinsky closure model to examine the boundary effect on the axisymmetric cavity with limited length over a disk or sphere in water tunnel of circular cross-section. He obtained an analytic solution which indicates that the flow could be blocked under some particular condition. This expanded the insight about boundary effects into the area of axisymmetric cavitating flows. Chen et al. [3] utilized the multiphase model based on Reynolds averaged Navier-Stokes equation system to numerically analyze the boundary effect on ventilated cavitating flows around a given submerged vehicle with a disk cavitator in closed water tunnel.

In addition, some other researches about the free surface effect on cavitating flows also have been done. Within the framework of the linearized theory, Franc and Michel [4] calculated the slope of the lift coefficient versus the angle of attack for cavitating hydrofoil with infinite cavities (cavitation number equals to 0 ) under a free surface and different immersion depths. For small or infinite value of the immersion depth, the slope tends towards the limited value.

However, the boundary effect on cavitating flows when a rigid wall and a free surface coexist has not been widely investigated yet so far. Bal and Kinnas et al. [5] extended 
the capability of the boundary element method [6-8] to computate cavitating flows around a hydrofoil in numerical wave tank, with the wall effect and free surface effect simultaneously taken into account. Amromin [9] employed a corrected Riabouchinsky closure model on the basis of ideal fluid theory to numerically analyze the shallow-water effect on supercavitating flow. The computational results indicated that for the same cavity length was reached, the cavitation number under the combination of a solid wall and a free surface is greater than that of axisymmetric flows, and the cross-section of cavity had some three-dimensional deformation.

Previous researches regarding boundary effects on cavitating flows were mostly limited in the area of potential flow theory, which has difficulty in describing the subtle structure inside the cavity and near the wake. Furthermore, viscous effects are impossible to be considered in the framework of potential flow. Thus, in the present work, numerical methods based on the viscous multiphase flow model [10-12] have been used to study boundary effects of the cavitating flow around two-dimensional wedge in shallow water.

\section{Governing Equations and Numerical Method}

In the present work, a single-fluid multiphase flow model based on the isotropic hypothesis for fluid and the Reynolds averaged Navier-Stokes equation will be employed. The mixture composed of air, vapor, and liquid is regarded as a kind of single fluid with variable density. Each component phase shares the same physical fields, namely, pressure, velocity, and so on. The gravity effect of liquid was considered, and the compressibility of gas was neglected. The volume fractions of air, vapor, and liquid phases, denoted as $\alpha_{a}, \alpha_{v}$, and $\alpha_{l}$, respectively, are introduced to obtain a set of governing equations describing the multiphase flow of air, vapor, and liquid phases.

The continuity equation for the mixture is as follows:

$$
\frac{\partial \rho_{m}}{\partial t}+\frac{\partial}{\partial x_{i}}\left(\rho_{m} u_{i}\right)=0
$$

The momentum theorem for the mixture holds as

$$
\begin{aligned}
& \frac{\partial}{\partial t}\left(\rho_{m} u_{j}\right)+\frac{\partial}{\partial x_{i}}\left(\rho_{m} u_{i} u_{j}\right) \\
& \quad=-\frac{\partial p}{\partial x_{j}}+\rho_{m} g_{i}+\frac{\partial}{\partial x_{i}}\left[\left(\mu_{m}+\mu_{t}\right)\left(\frac{\partial u_{i}}{\partial x_{j}}+\frac{\partial u_{j}}{\partial x_{i}}\right)\right] .
\end{aligned}
$$

The air phase should keep continuous as

$$
\frac{\partial}{\partial t}\left(\rho_{a} \alpha_{a}\right)+\frac{\partial}{\partial x_{i}}\left(\rho_{a} \alpha_{a} u_{i}\right)=0 .
$$

The vapor phase should also satisfy continuity during phase-transition process:

$$
\frac{\partial}{\partial t}\left(\rho_{v} \alpha_{v}\right)+\frac{\partial}{\partial x_{i}}\left(\rho_{v} \alpha_{v} u_{i}\right)=\dot{m}^{-}-\dot{m}^{+},
$$

where $\rho$ refers to the density $t$ is the flow time $u_{i}$ denotes each velocity component $p$ is the local pressure the subscripts $m, a, v$, and $l$, respectively, refer to the mixture, air, vapor and liquid phases $i$ and $j$ indicate the Cartesian coordinates which can be 1,2 , or 3 .

The volume fraction equation will not be solved for the primary phase, namely, the liquid phase. The volume fraction equation of liquid phase will be computed based on the following constraint:

$$
\alpha_{a}+\alpha_{v}+\alpha_{l}=1
$$

Additionally, two individual transport equations were employed to describe the mass-transfer process [13] as (4) between the vapor phase and the liquid phase:

$$
\begin{aligned}
& p<p_{v}, \quad \dot{m}^{-}=\frac{\rho_{l} \rho_{v}}{\rho_{m}} \alpha_{v}\left(1-\alpha_{v}\right) \frac{3}{R_{B}}\left[\frac{2}{3} \frac{p_{v}-p}{\rho_{l}}\right]^{1 / 2}, \\
& p>p_{v}, \quad \dot{m}^{+}=\frac{\rho_{l} \rho_{v}}{\rho_{m}} \alpha_{v}\left(1-\alpha_{v}\right) \frac{3}{R_{B}}\left[\frac{2}{3} \frac{p-p_{v}}{\rho_{l}}\right]^{1 / 2},
\end{aligned}
$$

where $R_{B}=\left(\left(\alpha_{v} /\left(1-\alpha_{v}\right)\right)(3 / 4 \pi n)\right)^{1 / 3}$ is the radius of vapor bubble, $n$ denotes the number of bubble nuclei per volume of liquid, and a value of $10^{12} / \mathrm{m}^{3}$ would be sufficient for modeling the phase transition.

The volume-fraction-averaged density and viscosity of the mixture take on the following forms:

$$
\begin{aligned}
& \rho_{m}=\alpha_{a} \rho_{a}+\alpha_{v} \rho_{v}+\alpha_{l} \rho_{l}, \\
& \mu_{m}=\alpha_{a} \mu_{a}+\alpha_{v} \mu_{v}+\alpha_{l} \mu_{l} .
\end{aligned}
$$

Moreover, the Reliable $k-\varepsilon$ turbulence model [14] together with the wall function [15] was used to calculate the turbulence viscosity, $\mu_{t}$, and make the governing equations closed.

The finite volume method was utilized to discretize the integral governing equation set, and the high-resolutioninterface-capturing high-order convection scheme [16] was employed to capture the free surface. In the equations, the temporal term was discretized with a first-order implicit scheme and second-order upwind scheme was employed for both the pressure term and the convection term. The convection term in turbulence model equation was approximated with first-order upwind scheme especially. The SIMPLE algorithm [17] was adopted to use a relationship between velocity and pressure corrections to enforce mass conservation and to obtain the pressure field. The ultimately linearized algebra equation system was solved with the Gauss-Seidel iteration method [18] and algebraic multigrid method. In computation, a time step of $10^{-4} \mathrm{~s}$ was specified to divide the totally physical time of $0.15 \sim 0.2 \mathrm{~s}$. The maximum of inner iterations in each time step was set to be 50 .

\section{Computational Domain and Boundary Conditions}

The 2D computational domain and boundary conditions are illustrated in Figure 1, where the length and the bottom 
width of the symmetric wedge are $c=60 \mathrm{~mm}$ and $D=$ $17 \mathrm{~mm}(R=0.5 D)$. The origin of the coordinate system is located at the tip of the wedge. The positive directions of $x$ and $y$ axes point rightward and upward, respectively. The inlet boundary and the outlet boundary are $60 c$ and $300 c$ away from the origin. The wedge has an immersion depth of $h=70,140,210 \mathrm{~mm}$, respectively. The rest free surface is of $H=280 \mathrm{~mm}$ high from the water bottom. The top boundary of the computational domain is $1.75 \mathrm{H}$ away from the bottom. The domain was divided into multiblock structured mesh that has 100800 cells.

The physical boundaries are assigned with suitable conditions. The inlet boundary and the top boundary were disposed with constant velocity $\left(u_{1}=0 \mathrm{~m} / \mathrm{s}\right.$ for $y>h$, or else $\left.u_{1}=10 \mathrm{~m} / \mathrm{s} ; u_{2}=0 \mathrm{~m} / \mathrm{s}\right)$. The outlet boundary was specified with fixed static pressure ( $p_{\text {outlet }}=P_{0}$ for $y>h$, or else $p_{\text {outlet }}=\rho_{0}-\rho_{l} g y$ ), which is used to adjust the cavitation number. The surface of the wedge and the bottom boundary were given no-slip conditions.

\section{Results and Discussion}

In this section, influence of boundary effects on several aspects including cavity patterns, free surface's shapes, and hydrodynamics of the wedge was discussed as follows.

4.1. Cavity Pattern. Figure 2 presents the typical cavity patterns with a free surface at some specific values of cavitation number, which is defined as $\sigma_{v}=\left(P_{\infty}-p_{v}\right) / 0.5 \rho_{l} V_{\infty}^{2}$. The cavity outline is defined as the interface where the isosurface of $\alpha_{v}=0.5$ locates. Here, $p_{v}$ indicates the saturated vapor pressure of water at specific temperature and the $P_{\infty}$ and $V_{\infty}$ denote the upstream pressure and velocity at inlet boundary on $y=0$.

It seems obvious that the size of the cavity becomes smaller if the cavitation number increases. According to contours of the volume fraction of water and the magnitude of the vorticity, cavity patterns can be roughly divided into the three types as follows.

(a) Steady Type: the cavitation number $\sigma_{v}$ is approximately in the range of $0.05 \sim 0.3$. The cavity presents the state of supercavity which is almost full of vapor. The cavity retains a stable shape except for some tiny oscillations near the tail of the cavity. The magnitude of the vorticity is much higher near the cavity surface, at the tailing part of the cavity, and behind the wedge.

(b) Transition Type: the cavitation number $\sigma_{v}$ is approximately in the range of $0.3 \sim 0.5$. The cavity presents the state of cloudy mixture composed of vapor and liquid, which is produced by the strong reentrant jet generated from the tail of the cavity. The cavity can roughly keep relatively clear outline. The magnitude of the vorticity is much higher in the band-shaped zones originated from the ends of the wedge's base.

(c) Wake-Vortex Type: the cavitation number $\sigma_{v}$ is approximately in the range of $0.5 \sim 2.04$. The cavity presents the state of vortex cavitation, which occurs in shedding vortices of the wake flow and exhibits an evident periodicity.
TABLe 1: Parameters for fitted curves of the free surface.

\begin{tabular}{lllll}
\hline$h / H$ & $\bar{h}_{\mathrm{fs} 0}$ & $A$ & $x_{c}$ & $w$ \\
\hline 0.25 & 0 & 1 & 0.48 & 0.309 \\
0.50 & 0 & 1 & 0.50 & 0.319 \\
0.75 & 0 & 1 & 0.52 & 0.325 \\
\hline
\end{tabular}

For the steady type of the cavity pattern, three relationships of cavity length $L_{c}$ with the cavitation number $\sigma_{v}$ is shown in Figure 3 where scattered symbols represent the numerical values and solid curves [4] were fitted from experimental data. Good agreement can be observed from the case of $h / H=0.25$, whereas the relative errors between the numerical and the experimental results get obvious for the other immersion depths. This fact could be due to the reason that the fitted curves of the experiments were obtained from the working condition of about $\sigma_{v}>0.1$ in the cases of $h / H>0.25$. These curves could not completely describe the present computational results in the range of $\sigma_{v}<0.1$ at the immersion depth of $h / H>0.25$.

For a fixed cavitation number, the cavity length is increasing when the immersion depth goes upward. The boundary effect of the solid wall is much stronger than that of the free surface, which enables the flow blocked to make the local cavitation number decrease and the cavity size enlarge.

4.2. Shapes of Free Surface and Cavity Interface. Because shapes of the transition type and the wake-vortex type of cavity are too unstable to be analyzed quantitatively, only the steady type of cavity was selected to be discussed in this section.

The height differences $h_{\mathrm{fs}}$ between the free surfaces at the conditions of deformation and of stillness are normalized as regard the maximal height difference $h_{\mathrm{fs} \text { max }}$, to obtain the universal free surfaces for three different immersion depths in Figures 4, 5, and 6 where the free surfaces are defined as the contour of $\alpha_{a}=0.5$.

It can be observed from the figures that the free surface's shapes of the cases are close to each other for $\sigma_{v}<$ $0.103(h / H=0.25), \sigma_{v}<0.109(h / H=0.50)$, and $\sigma_{v}<$ $0.116(h / H=0.75)$ respectively, approaching the curve of the Gauss function $\bar{h}_{\mathrm{fs}}=\bar{h}_{\mathrm{fs} 0}+A e^{-0.5\left(\left(\bar{x}-x_{c}\right) / w\right)^{2}}$. Here, $\bar{h}_{\mathrm{fs}}=$ $\left(h_{\mathrm{fs}}^{2}-\left(R^{2} / 4\right)\right) /\left(h_{\mathrm{fs} \max }^{2}-\left(R^{2} / 4\right)\right), \bar{x}=x / L_{c}$; the values of $\bar{h}_{\mathrm{fs} 0}, A, x_{c}$, and $w$ are listed in Table 1 . In order to satisfy the requirement of the normalization, the value of $A+\bar{h}_{\mathrm{fs} 0}$ must be one. The level of the free surface for the remained cases is relatively higher. In particular, the irregularity of the deformation of the free surface is more distinct when $\sigma_{v}$ is greater than about 0.25 .

The influence of the free surface is quite different from that of the bottom wall on the shape of the cavity. Thus, the upper and the lower surface of the cavity must be discussed, respectively.

Figures 7, 8, and 9 present the profiles of the upper surface of the cavity when the cavity width $R_{\text {up }}$ is normalized as to the maximal cavity width $R_{\text {up } \max }$, where the cavity 


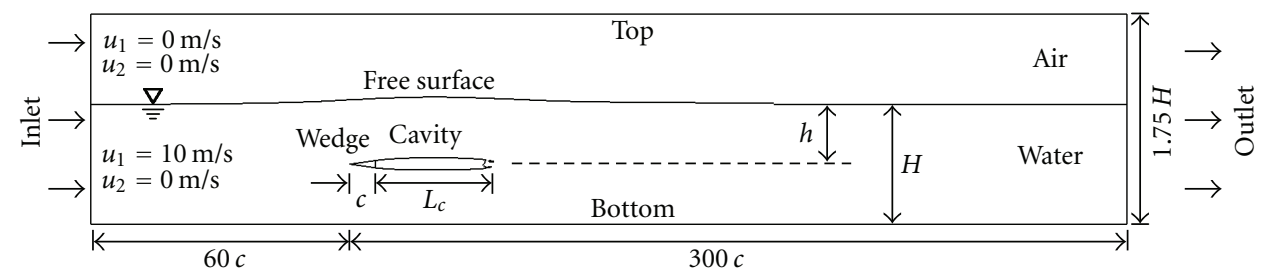

FIgURE 1: Computational domain and boundary specification.
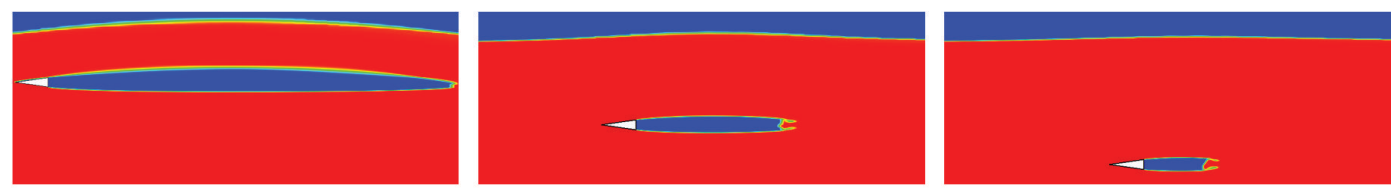

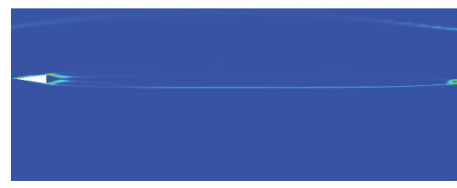

$h / H=0.25, \sigma_{v}=0.054$

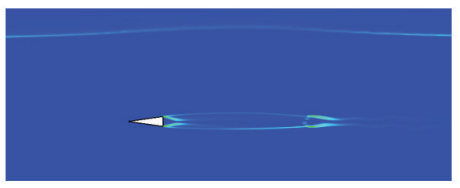

$h / H=0.5, \sigma_{v}=0.157$

(a)
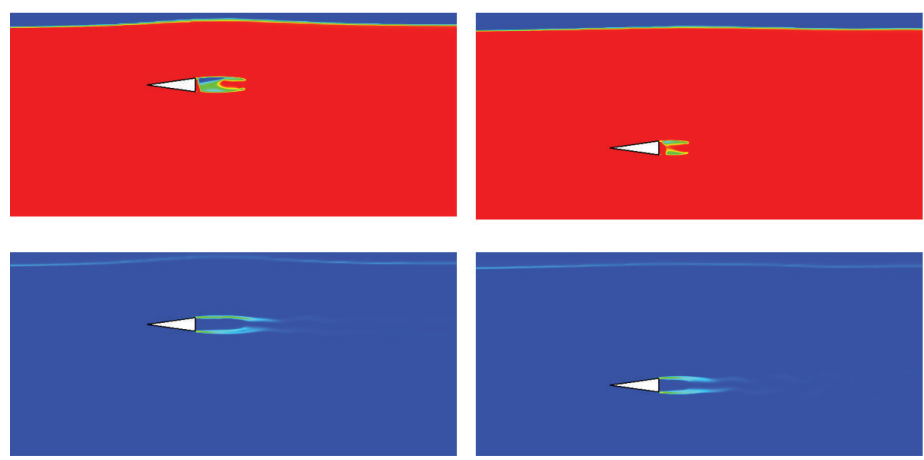

$h / H=0.25, \sigma_{v}=0.302$

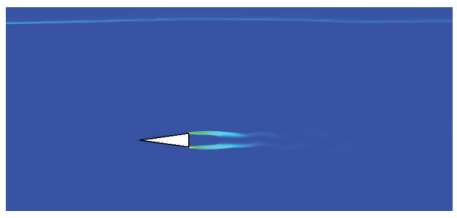

$h / H=0.5, \sigma_{v}=0.405$

(b)
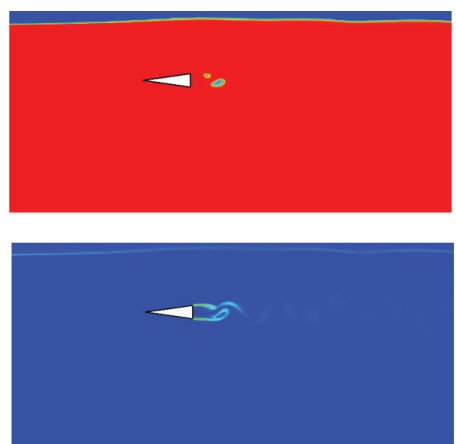

$h / H=0.25, \sigma_{v}=0.6$
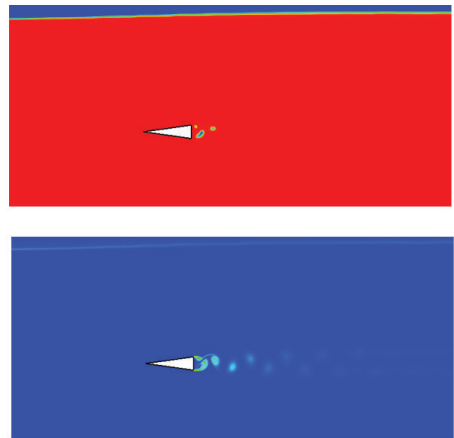

$h / H=0.5, \sigma_{v}=1.207$

(c)

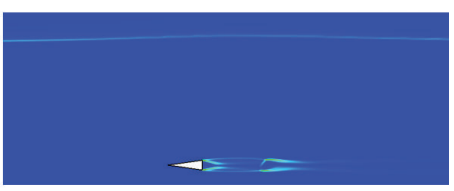

$h / H=0.75, \sigma_{v}=0.261$
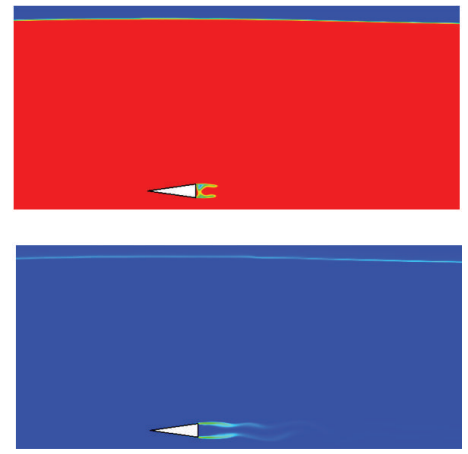

$h / H=0.75, \sigma_{v}=0.508$
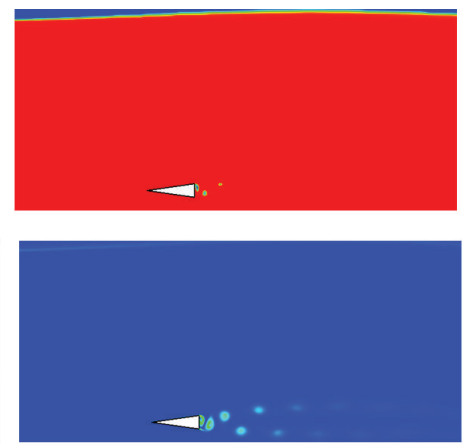

$h / H=0.75, \sigma_{v}=2.039$

FIGURE 2: Three cavity patterns: (a) steady type; (b) transition type; (c) wake-vortex type; the upper is the contour of the volume fraction of water; the lower is the contour of the magnitude of the vorticity. 


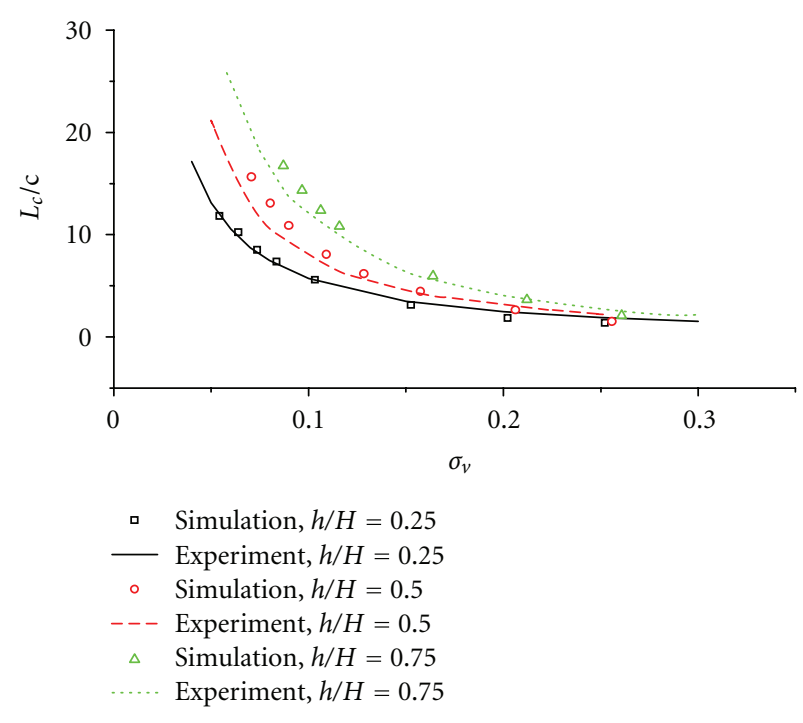

FIgURE 3: Relations between $L_{c}$ and $\sigma_{v}$.

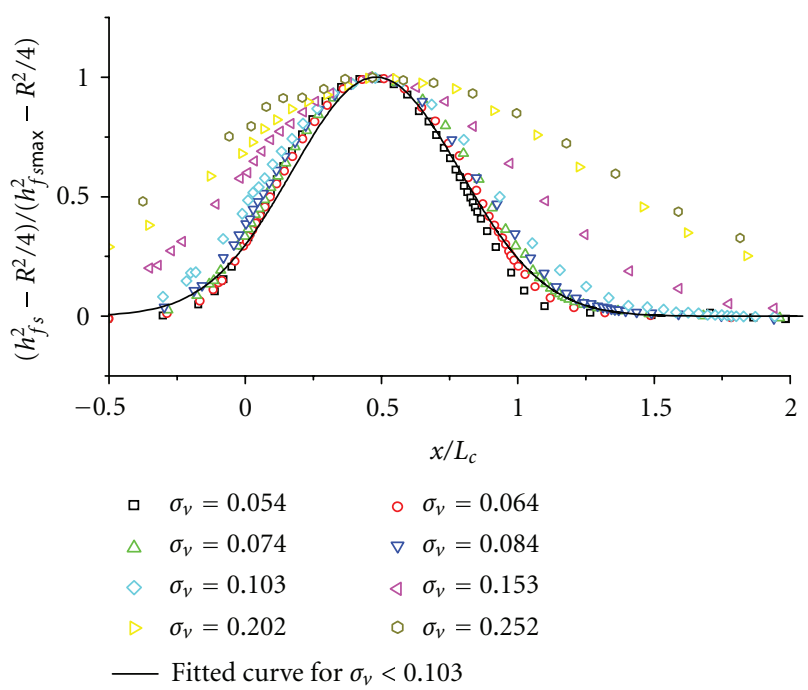

FIGURE 4: Universal profiles of the free surface at different $\sigma_{v}, h / H=$ 0.25 .

width is defined as the vertical length from any specific point on cavity surface to the symmetry line of the $2 \mathrm{D}$ wedge.

One can see that the upper surfaces of the cavity present pretty good unity in the cases for $\sigma_{v}<0.103(h / H=0.25)$, $\sigma_{v}<0.109(h / H=0.50)$, and $\sigma_{v}<0.116(h / H=0.75)$, respectively. However, for other cases, the width of the back part of the cavity is much bigger, with a concave distortion in the vicinity of the cavity closure because of the reentrant jet. By direct translating the fitted curves in Figures 4-6 to be compared with the scattered cavity profiles in Figure 79 , it was found that the shape of the free surface is similar to that of the upper surface of the cavity in the cavitation region. Such phenomenon owes to the flexibility of the free surface which deforms along with the development of cavity surface. All the parameters for the fitted curves in

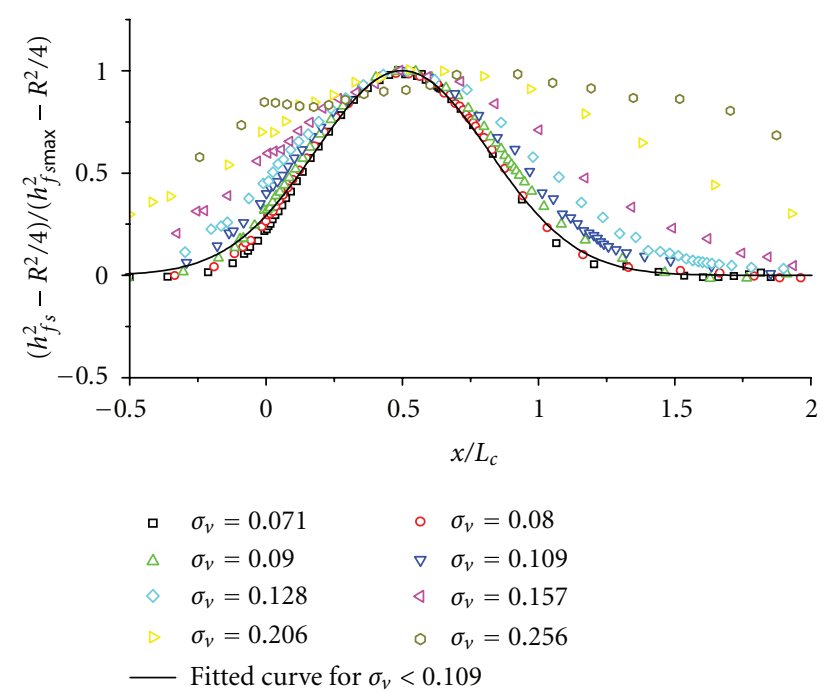

FIGURE 5: Universal profiles of the free surface at different $\sigma_{v}, h / H=$ 0.50 .

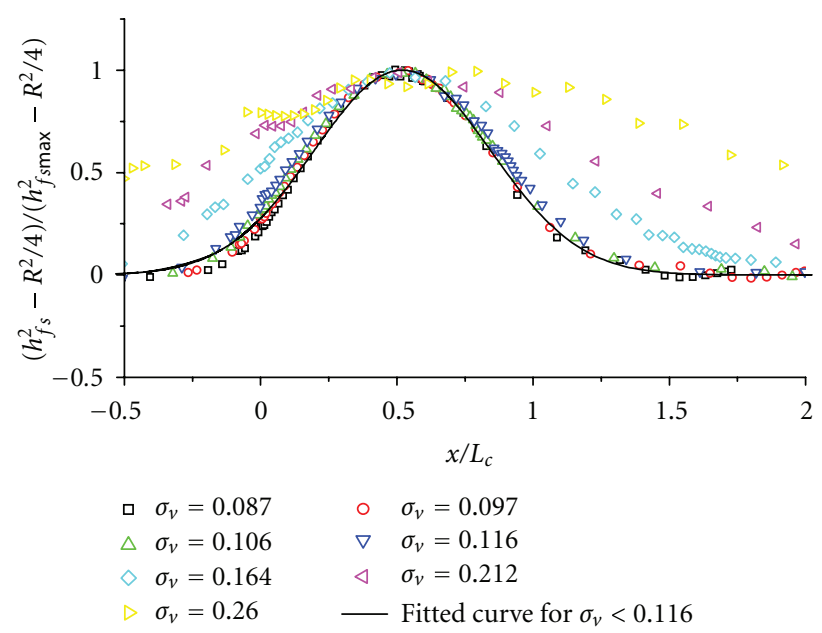

Figure 6: Universal profiles of the free surface at different $\sigma_{v}, h / H=$ 0.75 .

TABle 2: Parameters for fitted curves of the upper surface of the cavity.

\begin{tabular}{lllll}
\hline$h / H$ & $\bar{h}_{\mathrm{fs} 0}$ & $A$ & $x_{c}$ & $w$ \\
\hline 0.25 & -0.45 & 1.45 & 0.47 & 0.309 \\
0.50 & -0.42 & 1.42 & 0.50 & 0.319 \\
0.75 & -0.45 & 1.45 & 0.50 & 0.325 \\
\hline
\end{tabular}

Figures 7-9 are given in Table 2. Strictly, the shape of the cavity's upper surface is more appropriate to the curve of parabolic function. Here, the curves of Gauss function are adopted just for illustration of the similarity between profiles of the upper cavity surface and of the free surface.

Additionally, Figures 10, 11, and 12 present the profiles of the lower surface of the cavity. The lower cavity width $R_{\text {low }}$ is normalized as to the maximal lower width $R_{\text {low max }}$. 


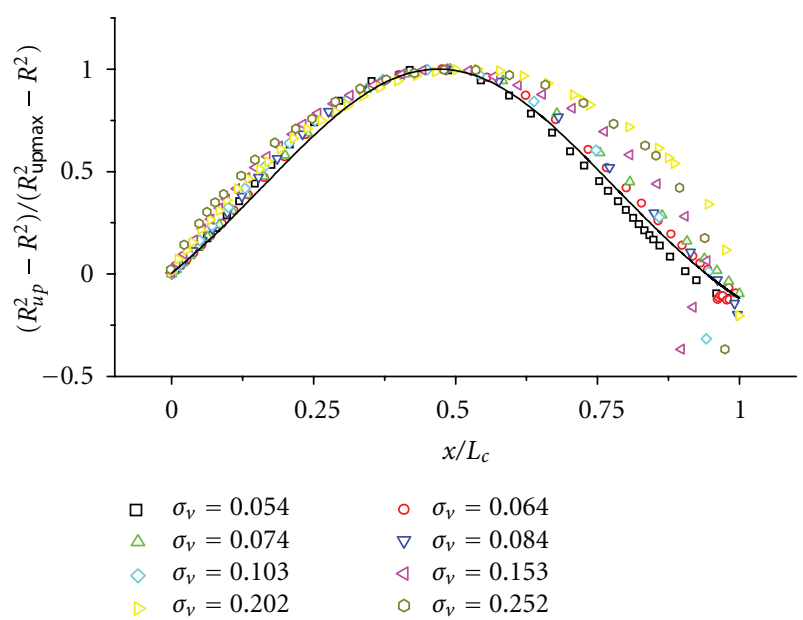

— Fitted curve of free surface for $\sigma_{v}<0.103$

FIGURE 7: Universal profiles of the upper surface of the cavity at different $\sigma_{v}, h / H=0.25$.

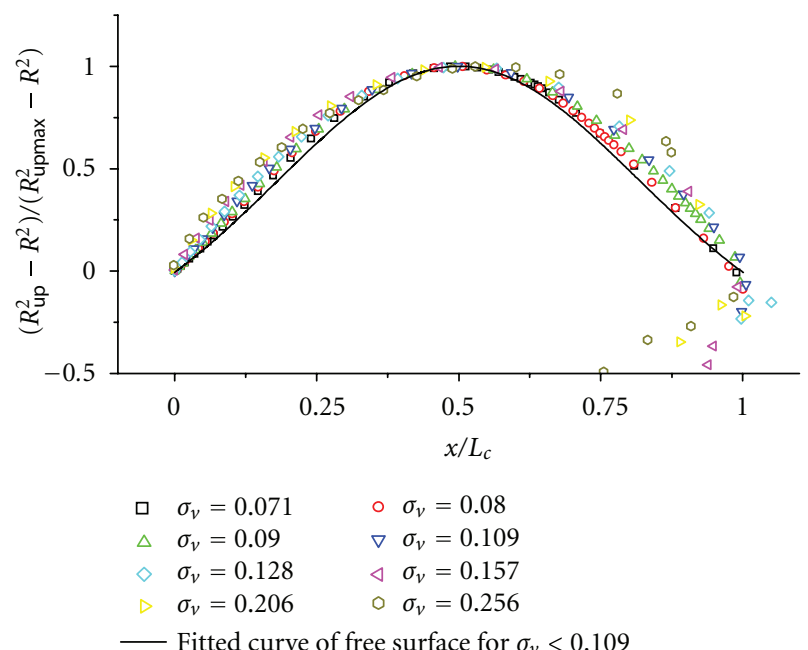

Figure 8: Universal profiles of the upper surface of the cavity at different $\sigma_{v}, h / H=0.50$.

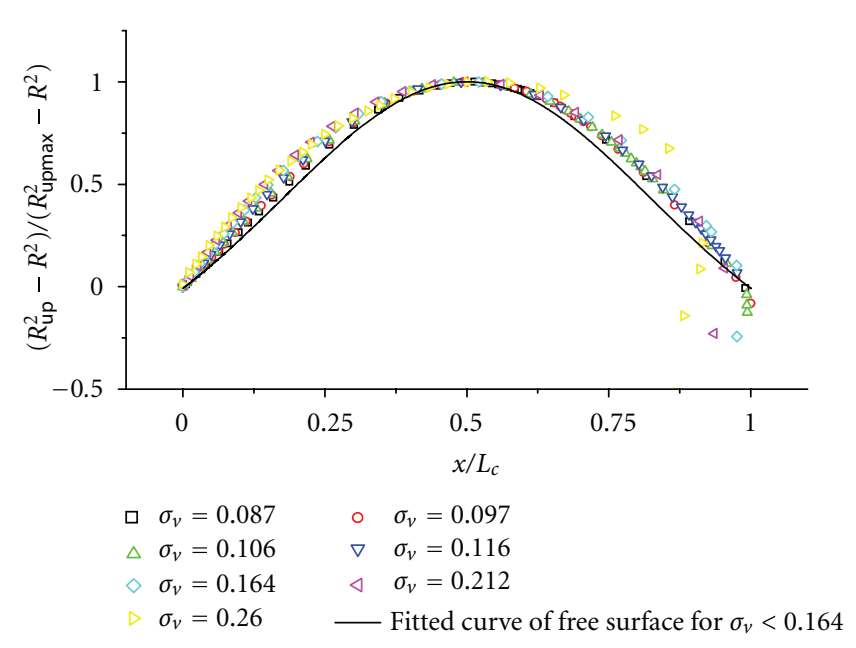

FIGURE 9: Universal profiles of the upper surface of the cavity at different $\sigma_{v}, h / H=0.75$.

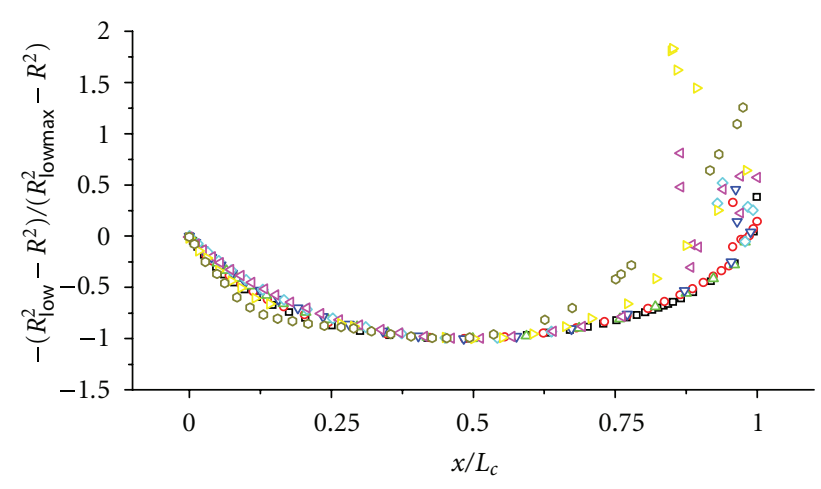
ㅁ $\sigma_{v}=0.054$
○ $\sigma_{v}=0.064$
$\triangle \sigma_{v}=0.074$
$\nabla \quad \sigma_{v}=0.084$
$\diamond \sigma_{v}=0.103$
$\triangleleft \sigma_{v}=0.153$
$\sigma_{v}=0.202$
○ $\sigma_{v}=0.252$

Figure 10: Universal profiles of the lower surface of the cavity at different $\sigma_{v}, h / H=0.25$.

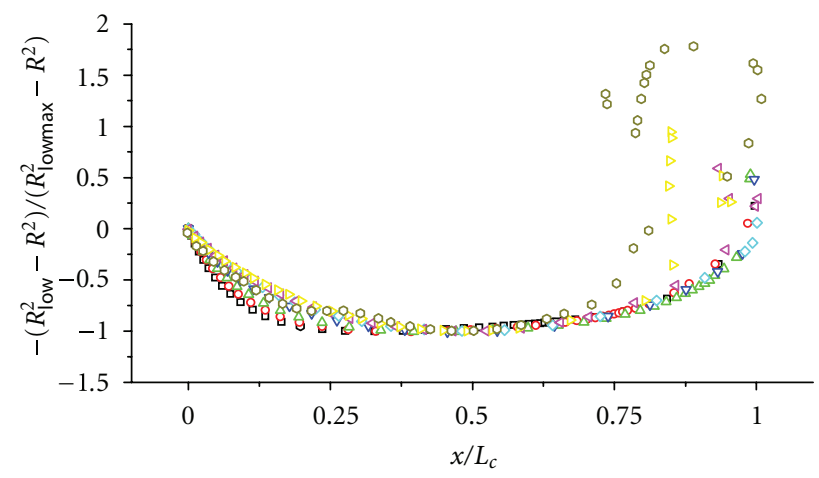
$\square \sigma_{v}=0.071$
○ $\sigma_{v}=0.08$
$\triangle \sigma_{v}=0.09$
$\nabla \sigma_{v}=0.109$
$\diamond \sigma_{v}=0.128$
$\triangleleft \sigma_{v}=0.157$
$\sigma_{v}=0.206$
$\sigma_{v}=0.256$

Figure 11: Universal profiles of the lower surface of the cavity at different $\sigma_{v}, h / H=0.50$.

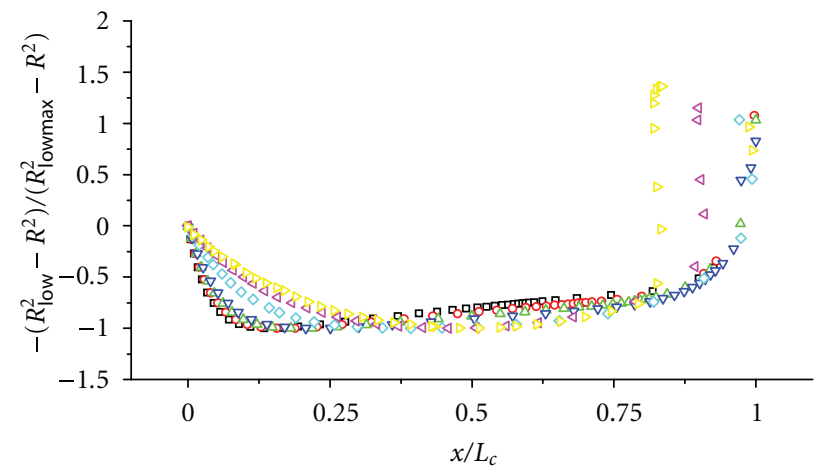
$\square \quad \sigma_{v}=0.087$
○ $\sigma_{v}=0.097$
$\triangle \sigma_{v}=0.106$
$\nabla \quad \sigma_{v}=0.116$
$\sigma_{v}=0.164$
$\triangleleft \sigma_{v}=0.212$
$\sigma_{v}=0.26$

FIGURE 12: Universal profiles of the lower surface of the cavity at different $\sigma_{v}, h / H=0.75$. 


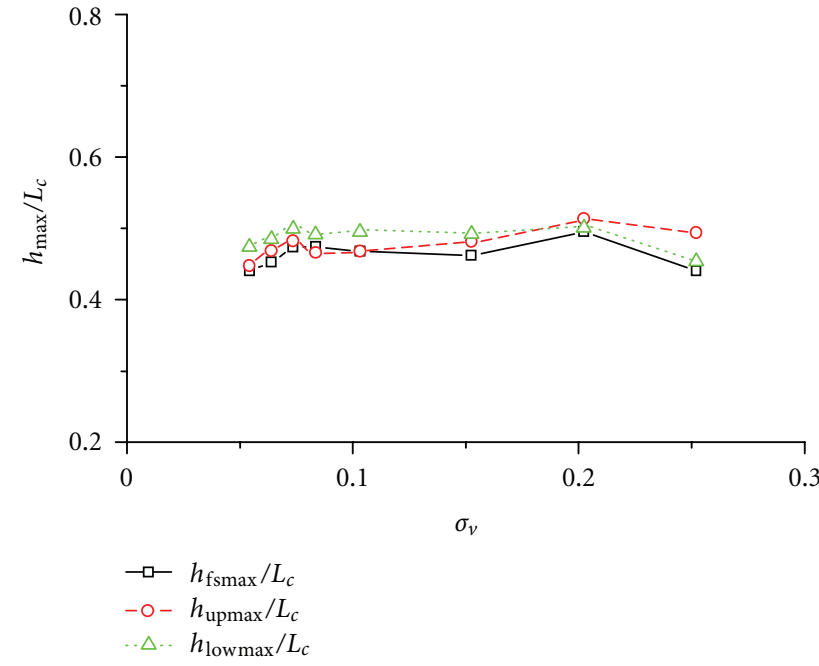

Figure 13: Positions located in the maximal level of the free surface and the maximal width of the cavity surface at different $\sigma_{v}, h / H=$ 0.25 .

A close observation shows the irregular deformation in the tailing part of the cavity in the cases for $\sigma_{v}>$ $0.202(h / H=0.25), \sigma_{v}>0.206(h / H=0.50)$, and $\sigma_{v}>$ $0.212(h / H=0.75)$ respectively, which results from the shedding and concavity of the cavity caused by reentrant jet. As a whole, the lower surface of the cavity has relatively poorer similarity with the increasing immersion depth. In the major part of the cavitating zone, the lower surface looks much flatter than the upper one. This is actually due to the existence of the rigid bottom wall, which prevents the lower surface of the cavity from developing in the vertical direction. Because there is a big difference in shapes of the cavity's lower surface, it is difficult that a normalized fitted curve can be given for each of the three immersion depths.

Figures 13, 14, and 15 present the relations between the cavitation number with positions located in the maximum of the level of free surface as well as the width of cavity surface.

For $h / H=0.25$, the position located in the maximal level of the free surface is very close to those in the maximal widths of the upper and lower surfaces of cavity. This indicates the shapes of the free surface and the cavity surfaces preserve symmetry. Along with the increase of the immersion depth, for $\sigma_{v}>0.2$ in the rough, the positions for the maximal widths of the upper and lower surface of cavity are close to each other, but the position for the maximal level of the free surface obviously moves backward. When the cavitation number $\sigma_{v}$ is roughly less than 0.2 , the position located in the maximal level of the free surface is still close to that in the maximal width of the cavity's upper surfaces, which implies that the profiles of the free surface and the cavity's upper surface maintain right-and-left symmetry. However, for $h / H=0.50$ and $\sigma_{v}<0.1$ or $h / H=0.75$ and $\sigma_{v}<0.2$, with the dropping $\sigma_{v}$, the position for the maximal width of the cavity's lower surface moves forward, which reveals that the up-and-down symmetry shape of the cavity surface is destroyed. By these geometrical results, one can see that the

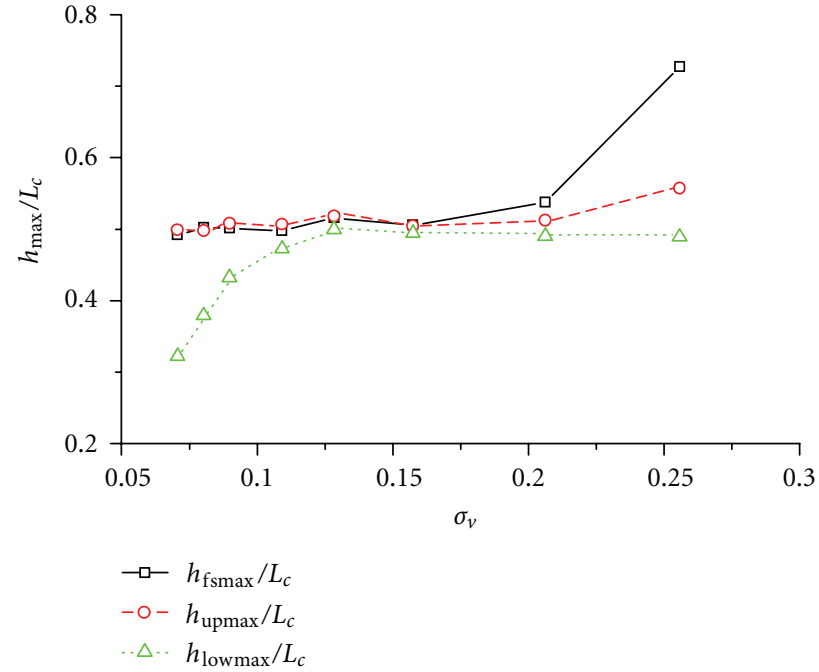

FIGURE 14: Positions located in the maximal level of the free surface and the maximal width of the cavity surface at different $\sigma_{v}, h / H=$ 0.50 .

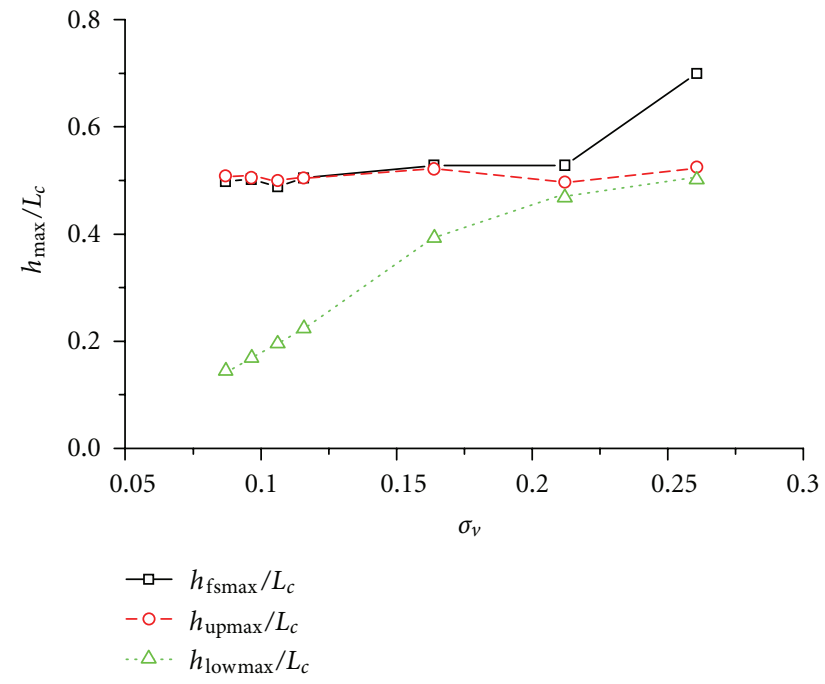

Figure 15: Positions located in the maximal level of the free surface and the maximal width of the cavity surface at different $\sigma_{v}, h / H=$ 0.75 .

influence of the rigid solid wall on the shape of the free surface and cavity interface is much stronger than that of the flexible free surface.

4.3. Hydrodynamic Coefficients. The instability of the cavity could also induce the oscillation of the hydrodynamic coefficients, taking curves, for example, as illustrated in Figures 16 and 17. So, we will still analyze the cases of steady type cavity in this section.

Figures 18 and 19 present the relations between the cavitation number $\sigma_{v}$ and the predicted drag and lift coefficients defined as $C_{d}=F_{x} / 0.5 \rho_{l} V_{\infty}^{2} c$ and $C_{l}=F_{y} / 0.5 \rho_{l} V_{\infty}^{2} c$, where $F_{x}$ and $F_{y}$, respectively, stand for the component of the 


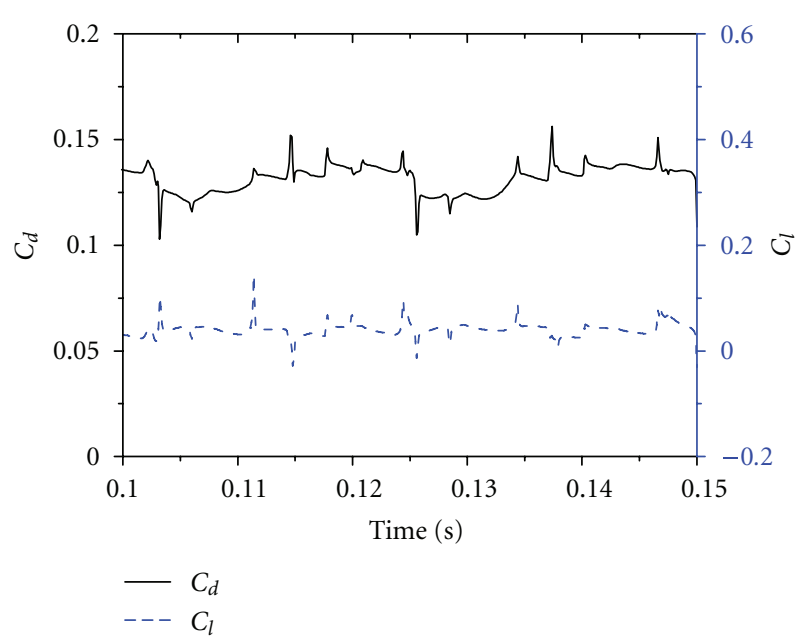

Figure 16: Variation of $C_{d}$ and $C_{l}$ along with $\sigma_{v}, h / H=0.50, \sigma_{v}=$ 0.507 , transition type cavity.

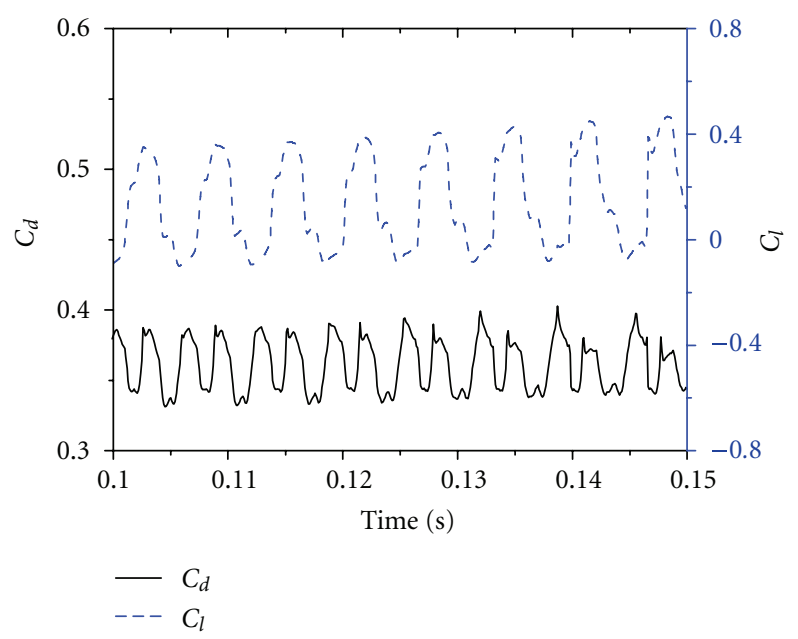

Figure 17: Variation of $C_{d}$ and $C_{l}$ along with $\sigma_{v}, h / H=0.75, \sigma_{v}=$ 1.808 , wake-vortex type cavity.

resultant force acting on the wedge in $x$ direction and $y$ direction.

It is clearly revealed from the curves that the drag coefficient becomes large gradually with an increase in cavitation number, whereas the lift coefficient curves describe a negative slope in the present computational range of the cavitation number. Furthermore, especially when the cavitation number is less than about 0.1 , the boundary effect of the free surface has an stronger influence on the drag coefficient for smaller immersion depth, whereas the boundary effect of the solid wall exerts an stronger influence on the lift coefficient for larger immersion depth. Such complicated influence on hydrodynamic coefficients mainly results from the different range of the cavitating zone and the displacement of the stagnation point near the tip of the wedge which is correlated to a change in circulation around the wedge [4].

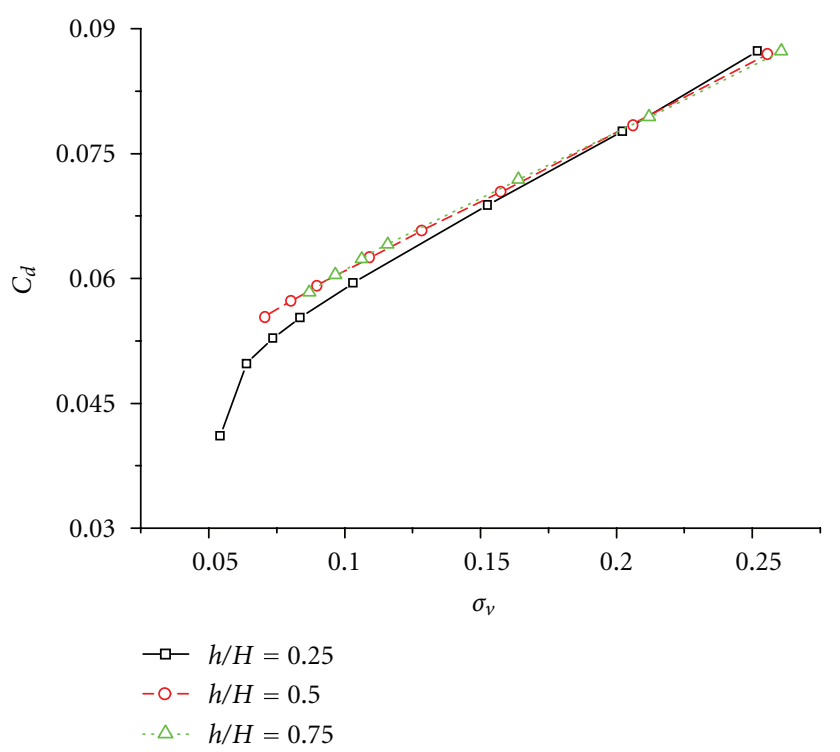

Figure 18: Relations between $C_{d}$ and $\sigma_{v}$.

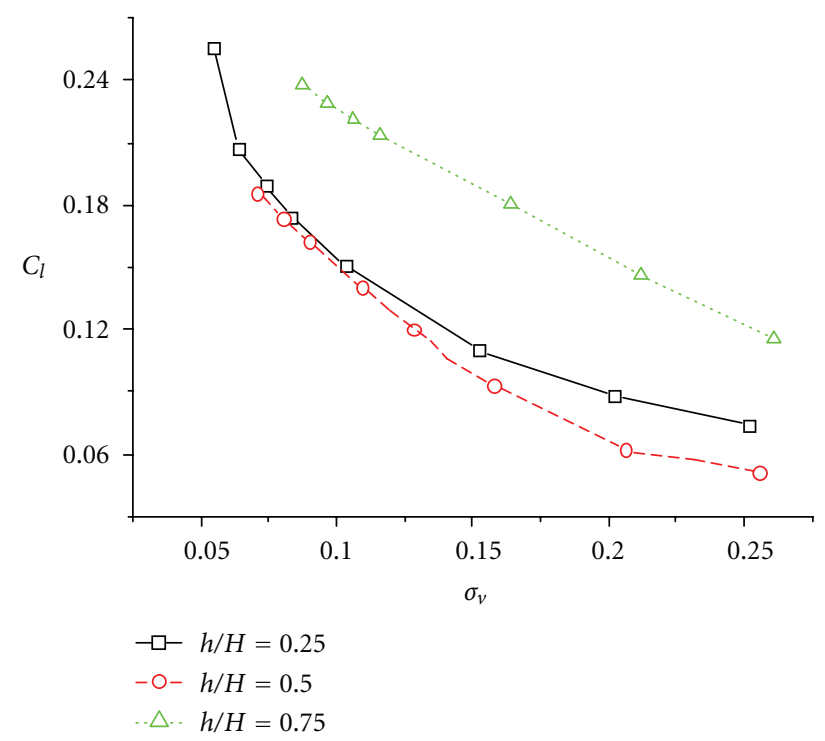

Figure 19: Relations between $C_{l}$ and $\sigma_{v}$.

\section{Conclusions}

In the present work, the natural cavitating flows around a two-dimensional wedge in shallow water were numerically investigated, by using the single-fluid multiphase flow model based on RANS equation coupled with a cavitation model. The influence of two types of boundaries on the cavity pattern, the shape of the free surface and hydrodynamic characteristics was studied. The following conclusions can be drawn by analysis and comparisons.

Firstly, in the range of the calculated cavitation number (0.05 2.04), cavity patterns can be divided into three types as steady type, transition type, and wake-vortex type. The successful simulation of the cavity of wake-vortex type 
demonstrates that the viscous multiphase model has an evident advantage over the numerical methods in traditional framework of potential flow.

In fact, the classes of cavity patterns here are still rough. Much more computation in a wider and finer range of cavitation number should be carried on to precisely determine the threshold value of cavitation number between two types of cavity pattern.

Secondly, for the cavity of steady type, the numerical results can be found to be quite in accordance with the experimental ones in the case of relative small immersion depth. But the deviation of the numerical values from the experimental data gets obvious for larger depths, especially at the cavitation numbers less than 0.1. Along with the reduction of the cavitation number, the cavity size increases as well as the deformation zone of the free surface. The free surface generally keeps consistent with the upper surface of the cavity in their shapes. However, the lower surface of the cavity looks much flatter due to the restriction of its growth in vertical direction by the effect of the rigid bottom wall. The position located in the maximal width of the cavity's lower surface moves forward gradually with the dropping cavitation number, which destroys the symmetry of the cavity shape.

Finally, the drag coefficient becomes large gradually with an increase in the cavitation number, while the lift coefficient curves describe a negative slope in the present computational range of cavitation number. Furthermore, when the cavitation number is smaller than about 0.1 , the boundary effect of the free surface has a stronger influence on the drag coefficient for smaller immersion depth, whereas the boundary effect of the solid wall exerts a stronger influence on the lift coefficient for larger immersion depth.

\section{Acknowledgments}

The Project was supported by the National Natural Science Foundation of China (Grant no. 11002089, 10832007) and the Shanghai Leading Academic Discipline Project (Grant no. B206). X. Chen is a Ph.D. Lecturer.

\section{References}

[1] G. Birkhoff, M. Plesset, and N. Simmons, "Wall effects in cavity flow," Quarterly of Applied Mathematics, vol. 8, article 161, 1950.

[2] C. E. Brennen, "A numerical solution for axissymmetric cavity flows," Journal of Fluid Mechanics, vol. 37, no. 4, pp. 671-688, 1969.

[3] X. Chen, C.-J. Lu, J. Li, and Z. C. Pan, "The wall effect on ventilated cavitating flows in closed cavitation tunnels," Journal of Hydrodynamics B, vol. 20, no. 5, pp. 561-566, 2008.

[4] J. P. Franc and J. M. Michel, Fundamentals of Cavitation, Kluwer Academic Publisher, Dordrecht, The Netherlands, 2004.

[5] S. Bal and S. A. Kinnas, "A numerical wave tank model for cavitating hydrofoils," Computational Mechanics, vol. 32, no. 4-6, pp. 259-268, 2003.
[6] S. Bal and S. A. Kinnas, "A BEM for the prediction of free surface effects on cavitating hydrofoils," Computational Mechanics, vol. 28, no. 3-4, pp. 260-274, 2002.

[7] S. Bal, S. A. Kinnas, and H. Lee, "Numerical analysis of 2-D and 3-D cavitating hydrofoils under a free surface," Journal of Ship Research, vol. 45, no. 1, pp. 34-49, 2001.

[8] S. Bal, "High-speed submerged and surface piercing cavitating hydrofoils, including tandem case," Ocean Engineering, vol. 34, no. 14-15, pp. 1935-1946, 2007.

[9] E. Amromin, "Analysis of body supercavitation in shallow water," Ocean Engineering, vol. 34, no. 11-12, pp. 1602-1606, 2007.

[10] A. K. Singhal, M. M. Athavale, H. Li, and Y. Jiang, "Mathematical basis and validation of the full cavitation model," Journal of Fluids Engineering, vol. 124, no. 3, pp. 617-624, 2002.

[11] X. Chen, C.-J. Lu, and L. Wu, "A multiphase model on simulating a ventilated cavitating flow," Journal of Hydrodynamics A, vol. 20, supplement, pp. 916-920, 2005 (Chinese).

[12] Y. Chen and C.-J. Lu, "A homogenous-equilibrium-model based numerical code for cavitation flows and evaluation by computation cases," Journal of Hydrodynamics B, vol. 20, no. 2, pp. 186-194, 2008.

[13] G. H. Schnerr and J. Sauer, "Physical and numerical modeling of unsteady cavitation dynamics," in Proceedings of the 4th International Conference on Multiphase Flow, New Orleans, La, USA, 2001.

[14] T. H. Shih, W. W. Liou, A. Shabbir, Z. Yang, and J. Zhu, "A new $\mathrm{k}-\varepsilon$ eddy-viscosity model for high Reynolds number turbulent flows-model development and validation," Computers \& Fluids, vol. 24, no. 3, pp. 227-238, 1995.

[15] E. Y. K. Ng, H. Y. Tan, H. N. Lim, and D. Choi, "Nearwall function for turbulence closure models," Computational Mechanics, vol. 29, no. 2, pp. 178-181, 2002.

[16] D. K. Walters and N. M. Wolgemuth, "An improved highresolution discretization scheme for volume-of-fluid CFD simulations," in Proceedings of the 5th Joint ASME/JSME Fluids Engineering Summer Conference (FEDSM '07), San Diego, Calif, USA, 2007.

[17] G.-G. Yang, X.-W. Ding, M.-S. Bi, and A. Abudula, "Improved SIMPLE algorithm used in numerical simulation of flammable gas cloud deflagration," Journal of Dalian University of Technology, vol. 44, no. 6, pp. 789-792, 2004 (Chinese).

[18] G.-H. Cheng, T.-Z. Huang, and X.-Y. Cheng, "Preconditioned Gauss-Seidel type iterative method for solving linear systems," Applied Mathematics and Mechanics, vol. 27, no. 9, pp. 12751279, 2006. 

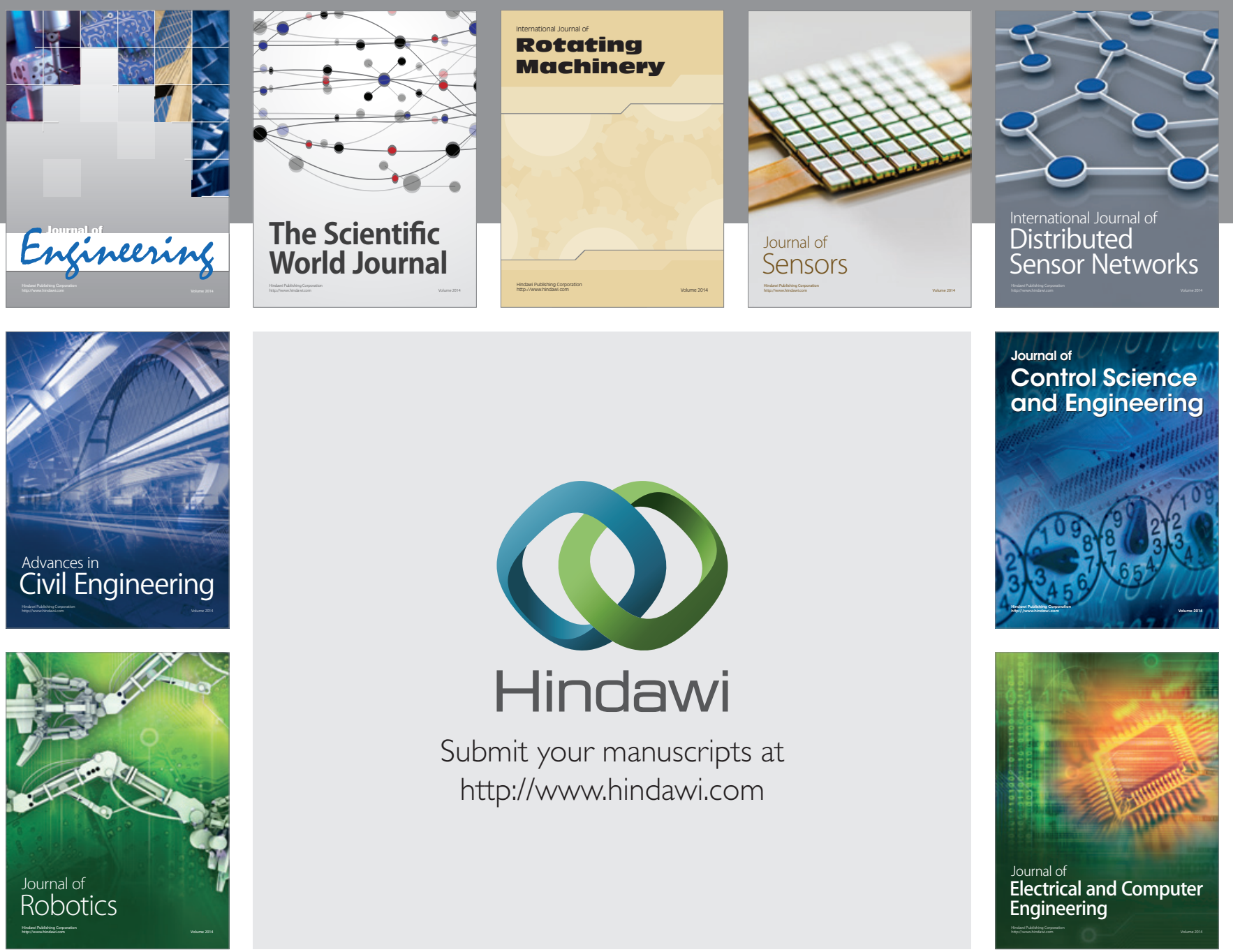

Submit your manuscripts at

http://www.hindawi.com
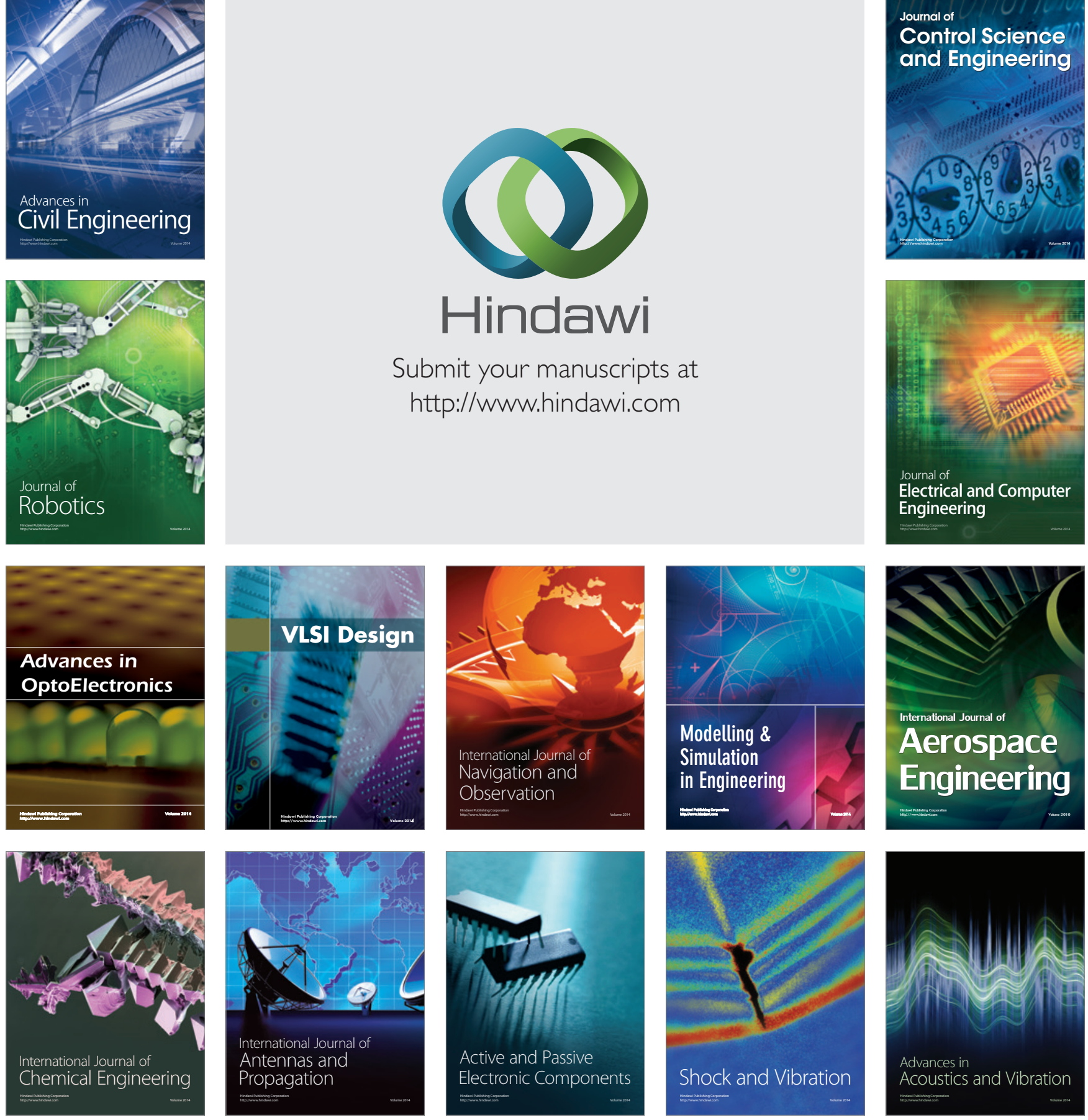